\title{
Optical strain gauge with high spatial resolution
}

\author{
L. Rodriguez-Cobo*, A. Cobo, and J.M. Lopez-Higuera \\ Photonics Engineering Group, University of Cantabria, Spain. \\ e-mail: luis.rodriguez@unican.es
}

January 21, 2014

\begin{abstract}
A technique to enhance the spatial resolution of typical FBG-based strain gauges has been experimentally verified in this work. Just analyzing the intensity of the reflection spectrum of a Sampled Fiber Bragg Grating (SFBG), its inner deformation profile has been obtained with a spatial resolution of $\Delta L<1 \mathrm{~mm}$. The proposed method employs a synthesis algorithm to retrieve the longitudinal axis strain profile of a SFBG glued to an asymmetric plate. The achieved experimental results have been compared to the mechanical simulations of the employed plate, exhibiting a good response even without compensating the residual stress provoked during the installation process.
\end{abstract}

Keywords: Strain sensor, Fiber Bragg Grating, High resolution, Finite Element Analysis, Spectral Synthesis

\section{Introduction}

Optical fiber sensors [1] have been widely applied to measure a wide set of different measurands on materials and structures [2]. Particularly, Fiber Bragg Gratings based transducers (FBGs) highlight over other optical techniques when measuring mechanical deformations [3]. Their multiplexing capabilities, where several sensing points can be concatenated in a fiber to give rise to quasi-distributed fiber sensors, enable this technology to monitor large working structures such as bridges $[4,5]$ and even to be employed in biomedical applications [6]. 
However, for most of the reported applications, each sensing point (FBG) is reported as the integrated measurand value along the whole FBG structure. This kind of transducers have a typical length of few millimeters, that works for many scenarios but, sometimes, the axial strain distribution within the FBG structure (with a given resolution) can be required. Several approaches have been proposed to obtain the deformation profile along the FBG structure, but most of them require phase measurements of the reflection spectrum $[7,8]$ or a-priori knowledge of the strain distribution [9-11]. These requirements increase the complexity of the sensor system, reduce the final dynamic response and, consequently, limit its use in potential real applications.

In this work, a FBG-based strain gauge has been improved to retrieve its inner deformation profile. Employing Sampled Fiber Bragg Gratings (SFBGs), a particular type of FBGs [12], in combination to a spectral synthesis technique [13], a sensor system has been developed and experimentally verified to retrieve the deformation profile of an asymmetric plate. Using only the intensity of the reflection spectrum of the proposed SFBG transducer, its intra-grating strain profile can be obtained, achieving an improvement on the spatial resolution of optical strain gauges. This improvement can be useful to a better characterization of advanced materials (e.g. composites) during their manufacturing process.

The proposed strain gauge has been properly attached to an asymmetric plate (mechanically modeled using Finite Element Analysis) to verify the suitability of the proposed sensor system. Measured and simulated strain profiles have been compared to verify the proposed sensor. Their very high correlation for different loading conditions validates the capability of the proposed scheme to obtain any axial strain profile with a given resolution. With the proposed transducer a spatial resolution of $\Delta L=0.7 \mathrm{~mm}$ was obtained using a SFBG of $N=7$ sections.

\section{Optical strain gauge: Fiber Bragg Grating}

In a simple way, a Fiber Bragg Grating (FBG) [14] is a periodic variation of the refractive index in the optical fiber core that reflects specific wavelengths. The reflected wavelengths are centered around the Bragg wavelength, defined by $\lambda_{\text {Bragg }}=2 n_{\text {eff }} \Lambda$, where $n_{\text {eff }}$ is the effective index (constant) in the fiber core and $\Lambda$ is the period of the refractive index variation. By elongating the FBG, $\Lambda$ is increased and, therefore, also the central wavelength $\left(\lambda_{B r a g g}\right)$. So, by measuring the central wavelength, the strain of the holder structure where the FBG is attached can be determined. This principle has been widely used 
for structural health monitoring in different fields such as civil engineering or renewable energies [3].
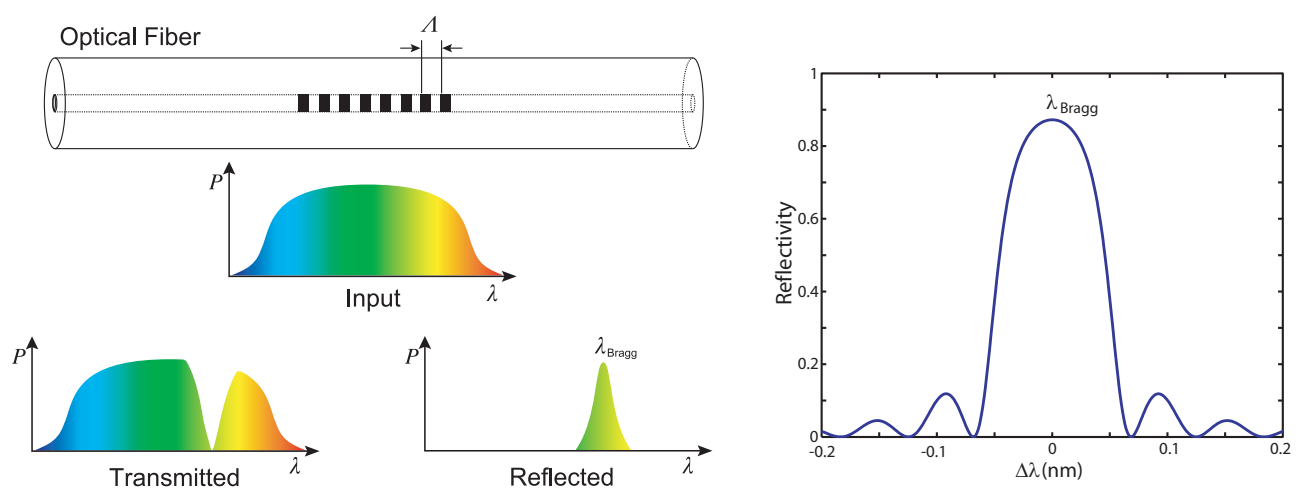

Figure 1: Basis of Fiber Bragg Grating (left) and a typical reflection spectrum of a non-apodized uniform FBG (right).

These periodic structures can be modulated, causing different artifacts detectable in their reflection spectrum. One possible modulation is to vary the amplitude of this periodic structure along the FBG length, achieving an "apodized" FBG [15]. This kind of modulation changes the shape of the main spectral peak and it can be employed to reduce the final noise or to generate ghost FBGs as happens in Sampled FBGs.

\subsection{Sampled Fiber Bragg Grating (SFBG)}

A Sampled FBG can be understood as a FBG whose axial index variation profile is modulated by a periodic sampling function of period $\mathrm{P}=2 \cdot \Delta L$ (Fig. $2)$. The period $(\mathrm{P})$ of the sampling function is much larger than the period of the grating, causing a double modulation in the structure: a rapidly varying component (of period $\Lambda$ ) and a slowly varying sampled envelope with a period $\mathrm{P}$. Based on Fourier theory, the periodic sampling function of period $\mathrm{P}$ can be expressed as a comb function modulated by complex coefficients. For each comb of the Fourier equivalent function, one ghost grating appears on the resulting spectrum. The spectral separation between two ghost grating is inversely proportional to the sampling period. A Sampled FBG has been chosen for this application because its spectral response exhibits particular artifacts that allow an easier comparison (such as different peaks).

Each FBG structure has its associated reflection spectrum. Particularly, an uniform FBG (as depicted in Fig.1, right) has a "Gaussian" similar spectral shape, that is replicated for each of the Fourier modes in a Sampled FBG 

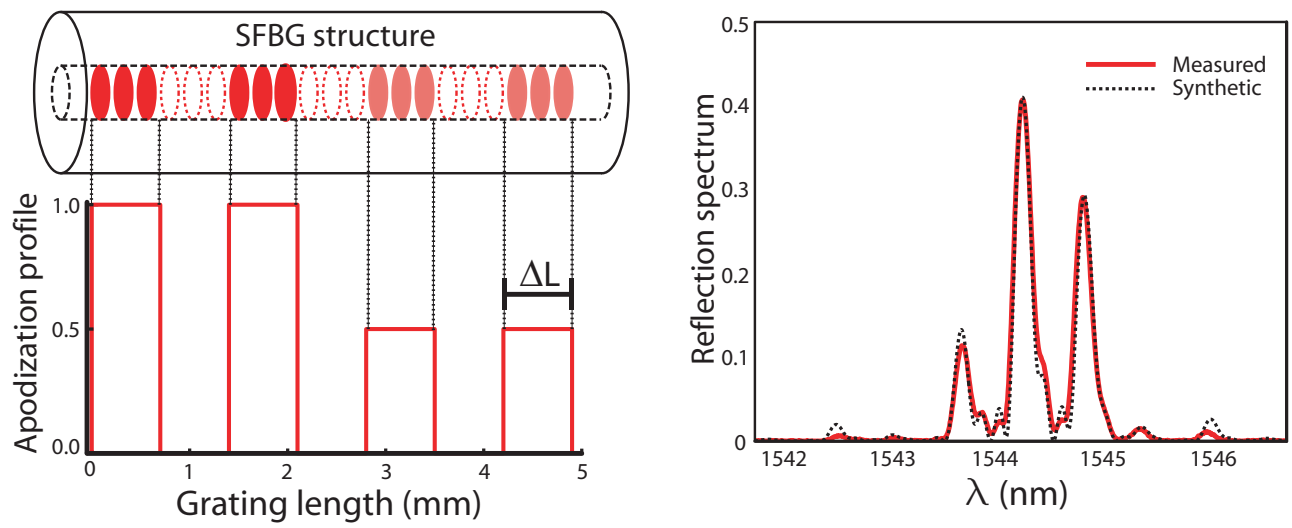

Figure 2: Illustration of one of the SFBG asymmetric structure and its reflection spectrum: the experimentally obtained and the one synthetized with the proposed technique. Some asymmetries were introduced to the apodized sampling function.

(Fig. 2, right). However, when an asymmetric strain is applied to the FBG period, its associated spectrum gets deformed, complicating its interrogation.

The SFBG structure is the basis of the spatial strain discrimination in this application. However, an asymmetric apodizing function is required to determine the direction of the strain vector. If a symmetric structure is employed, the synthetic spectrum will match under the same strain vector applied in opposite directions (forward and backward) because each SFBG section will contribute in the same way as its reciprocal does. If there are no other means to solve this uncertainty (i.e. having an a-priori knowledge about the strain profile), an asymmetrical sampling function must be used to avoid this problem. Fig. 2 (left) shows one of the employed asymmetric sampling functions of the SFBG. Each SFBG has $N=7$ sections of $\Delta L=0.7 \mathrm{~mm}$ length. The apodization value of the two first sections was set to "1" and the other two were set to "0.5". With this asymmetry, the first SFBG section contributes to the reflection spectrum in a different way than the last one, thus solving the direction uncertainty. The number of sections $(N)$ is mainly limited by the computation time and the spatial resolution $(\Delta L)$ must be set taking into account the expected deformation profile. 


\subsection{Spectral Synthesis}

Since the individual deformation of each section of a SFBG can be different, its spectral response has to be analyzed to extract its strain distribution along the FBG. Based on a synthetic algorithm, the structure of the FBG employed as strain gauge is simulated (in this case, $N=7$ sections of $\Delta L=0.7 \mathrm{~mm}$ length). Different deformation profiles are applied to the simulated model until its simulated spectrum matches the measured one.
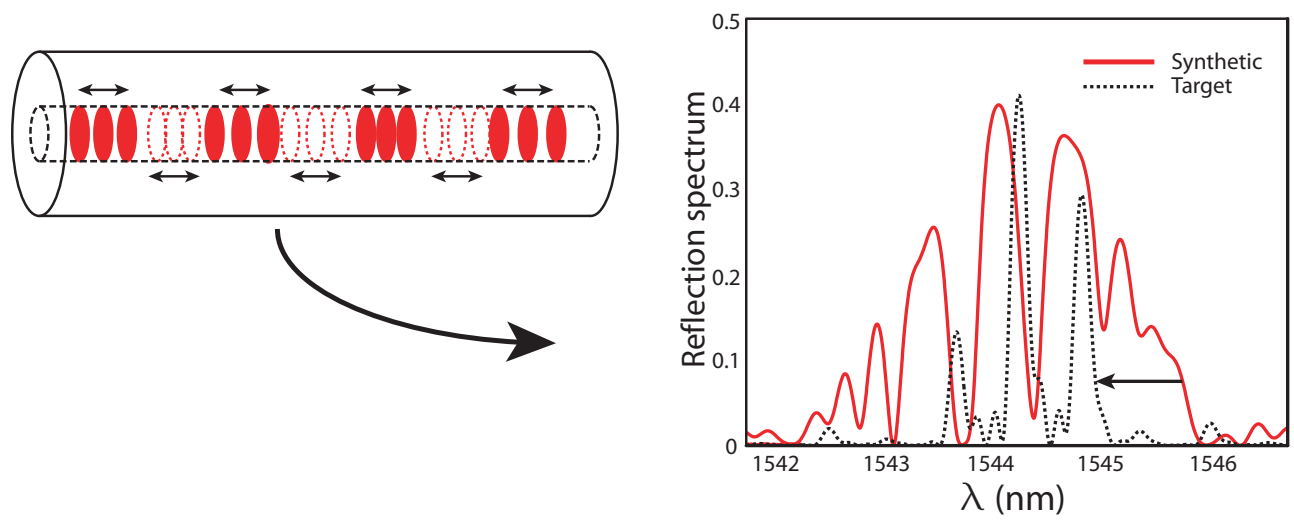

Figure 3: Different strain profiles are applied to the simulated model until its reflection spectrum matches the measured (target) one.

The measured spectrum is compared to simulated (synthetic) ones until they match [13] to get the deformation axial profile of a SFBG. Synthetic spectra are computed using the same SFBG base structure, but being artificially stretched (Fig. 3). Each SFBG section has a different value that corresponds to the averaged strain value over the section length $(\Delta L)$ so, the spatial resolution is given by the sampling period of the SFBG. The ultimate goal is the obtention of an artificial strain profile that, applied to the SFBG base structure, gives a synthetic spectrum that "matches" the measured one. This is a $N$-dimensional blind optimization problem where $N$ is the number of sections, and, Particle Swarm Optimization (PSO) [16] is employed to explore the different solutions. The PSO algorithm is combined with a custom defined error metrics [13] to evaluate the error between the measured and the synthetic spectra. 


\subsection{Sensor system}

The spectral synthesis can be combined to achieve a quasi-distributed sensor, as happens with standard FBGs. A scheme of the sensor system is depicted in Fig. 4. A white light source is used to interrogate the transducer through a circulator. The reflective transducer response is then launched into an Optical Spectrum Analyzer (OSA) through the optical circulator. The spectrum is acquired and computer-processed using the mentioned spectral synthesis technique, obtaining the strain distribution along the grating.

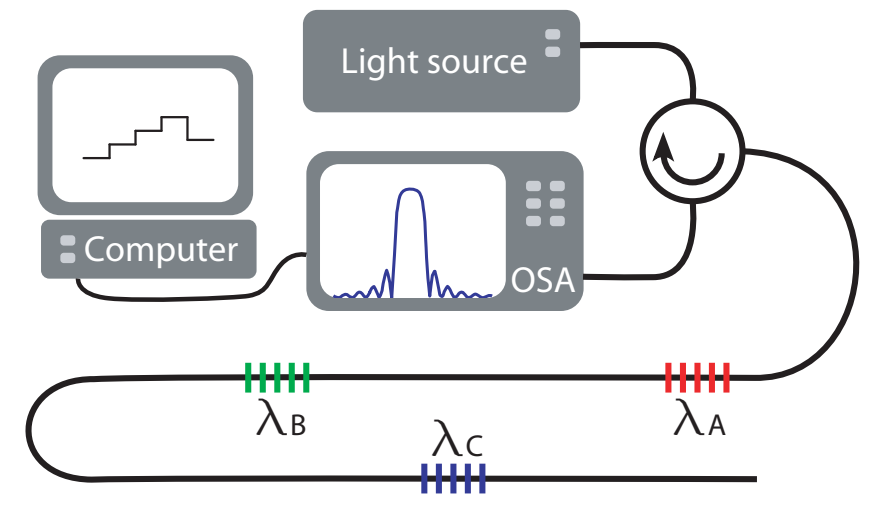

Figure 4: The fiber transducer is connected to an Optical Spectrum Analyzer (HP86140A) and to a light source (HP83437A) through a circulator. After the processing of the SFBG reflective response, the grating strain axial profile is obtained.

The spectrum of each sensing point (SFBG) is captured and processed using the mentioned technique obtaining a N-dimensional strain vector (being $N$ the number of sampling sections of the SFBG), which produces the intragrating strain profile. This process is repeated for each of the different sensing points to get the whole transducer response. Trying to analyze the performance of each sensing point, a single SFBG has been manufactured and experimentally tested.

\section{$3 \quad$ Experiments}

The SFBG was manufactured in a standard telecommunications optical fiber with the phase mask technique using a continuous laser emitting at $244 \mathrm{~nm}$. The photosensitivity of the fiber was increased with the Hydrogen loading technique. The sampling effect was generated by opening and closing the laser shutter at given locations. The apodization effect was generated by 
changing the recording speed during the laser scanning process: the higher the envelope, the lower the speed. The measured spectrum of the employed SFBG is depicted in Fig. 2 with the obtained synthetic spectrum from which the reference strain vector can be obtained to compensate the final result.

To check the capability of this proposed sensor system to reproduce nonuniform axial deformation profiles, the manufactured SFBG was installed in a plastic (PMMA) asymmetric plate to measure its longitudinal axis deformation. This plastic piece was manufactured using a CNC cutting machine. The SFBG was carefully glued in the middle axis of the piece using cyanoacrylate. In order to avoid the compensation step, the SFBG is preloaded with a homogeneous strain $(\Delta S \approx 400 \mu \epsilon)$ before its gluing process. With this step, the asymmetry of the residual stress due to the gluing process is minimized, simplifying the installation of the transducer. The asymmetric plate has been mechanically modeled (Fig. 5) using the Finite Element Analysis suite of the Autodesk Inventor (Autodesk Inc.), exhibiting a decreasing strain profile. Before the stretching of the plastic piece and to check the residual strain, the spectrum (Fig. 2) is captured and processed. The obtained strain vector falls within a convergence margin over the zero deformation, so further measurements do not have to be compensated. The convergence margin was empirically obtained $(\Delta s \approx 100 \mu \epsilon)$ by processing several times different spectra and obtaining their strain vector deviation mean.

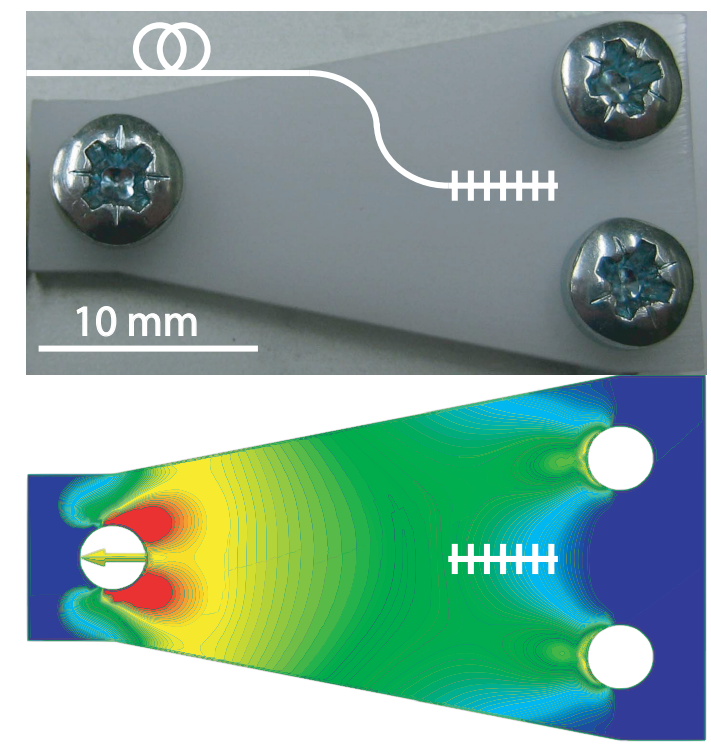

Figure 5: Plastic asymmetric plate and its mechanical stretching response.

The asymmetric modeled plate was stretched with a precision screw, leading the piece to different loads while the SFBG spectra were captured. These 
spectra were processed obtaining their synthetic spectra and strain vectors. The spectrum corresponding to the highest achieved deformation $\left(S_{1}\right)$ is depicted in Fig. 6 against its associated synthetic spectrum. As can be observed, both spectra are in very good agreement, exhibiting the same characteristic shape (two low peaks at lower wavelengths followed by two higher ones with a small side lobe in the middle). However, there are still small differences created by small contributions of several factors such as: the nonconstant strain value within the same SFBG section; slight differences in the gluing process; or even the interpolation technique and the resolution of the Optical Spectrum Analyzer (60 pm for the HP86140A) which reduce the correlation with the synthetic spectrum (not interpolated).

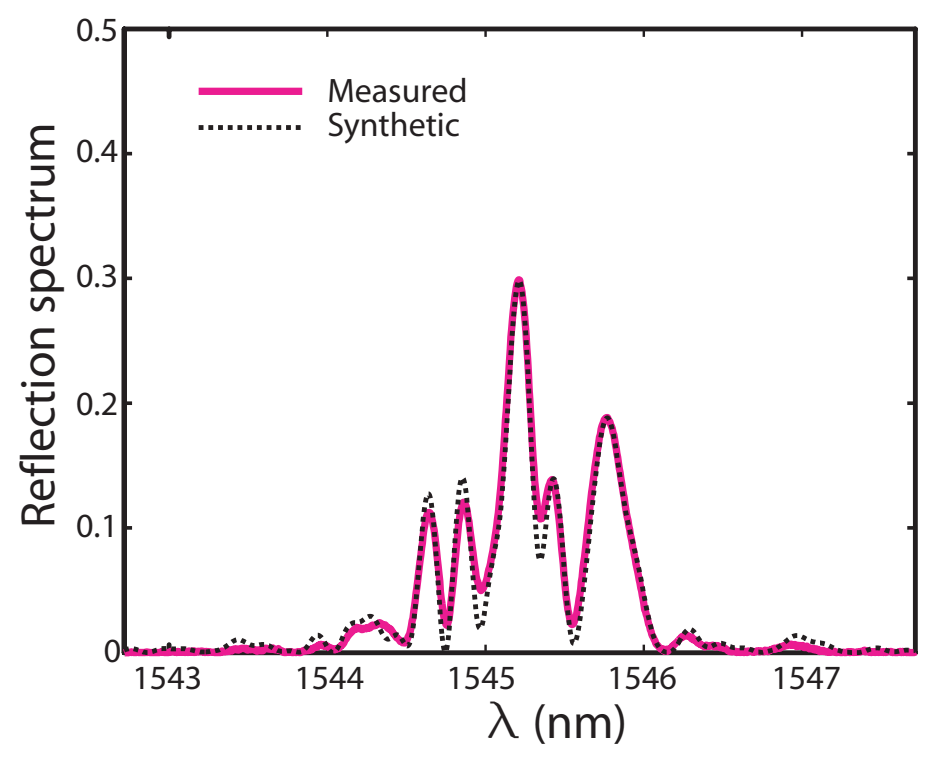

Figure 6: Measured (solid line) and synthetic (dotted line) spectra corresponding to the highest load.

\section{Results and discussion}

Several runs were performed for each of the captured spectra to get the convergence range (empirically obtained $\Delta s \approx 100 \mu \epsilon$ ). In Fig. 7 , several runs of two spectra are plotted against the scaled value of the FEA simulations. The two spectra are associated with two different loads (with mean deformation of $S_{1} \approx 250 \mu \epsilon$ and $S_{2} \approx 450 \mu \epsilon$ respectively). The spectral synthesis technique exhibits a decreasing strain profile as the SFBG section position is increased, obtaining the same decreasing slope for both tested loads. 


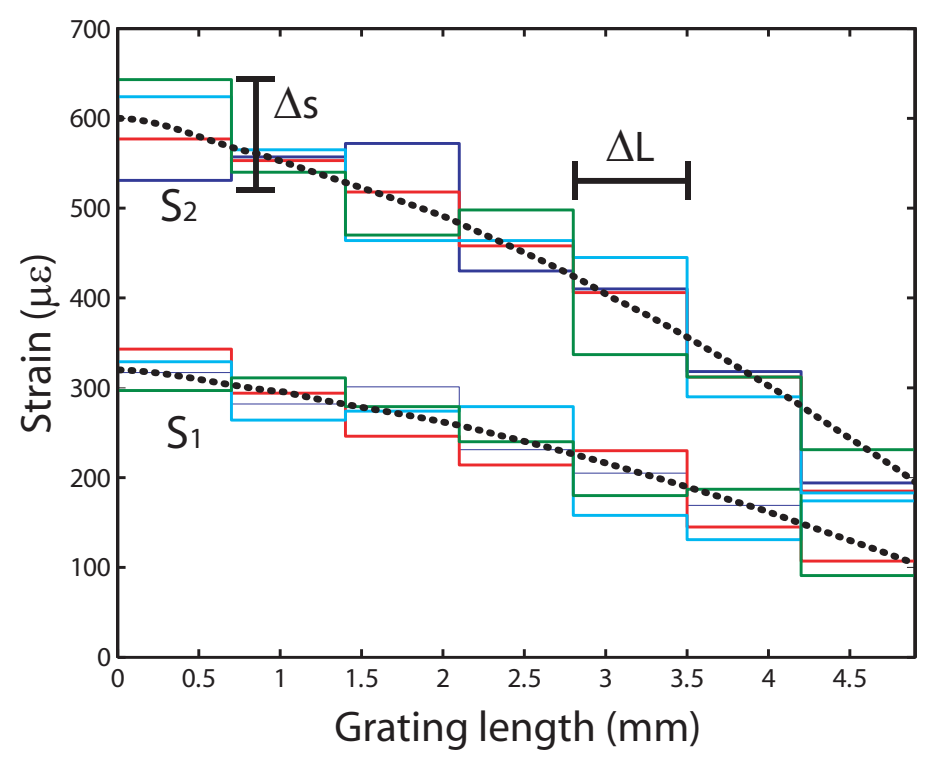

Figure 7: Different strain vectors obtained from two captured spectra (solid lines) against the scaled value of the FEA simulations (dotted lines). All the obtained strain profiles fall within the convergence range $(\Delta s)$

In this particular experiment, the gluing process has been performed with special care, preloading the SFBG to minimize the asymmetry of the residual strain profile to skip the compensation step (the non-loaded reference strain profile falling within the convergence range). Paying special attention to the installation process, the compensation step can be omitted thus simplifying the installation process. However, to improve the strain resolution, the initial strain profile can be employed to compensate the achieved solution.

The achieved convergence range can be excessive for some applications $(\Delta s \approx 100 \mu \epsilon)$ but it can be reduced by averaging different strain profiles (of the same spectrum). The spatial resolution $(\Delta L)$ is fixed by the sampling period of the SFBG and it can be also improved by using a lower sampling period. With a lower sampling period, a higher number of sections $(N)$ is required to cover the same length. Both techniques to improve the final resolution (strain and spatial) have an effect on the computation time and limit the final application of this sensor to quasi-static scenarios.

\section{Conclusion}

In summary, an optical strain gauge with improved spatial resolution has been experimentally verified. Based on a surface glued Sampled Fiber Bragg 
Grating, different strain profiles have been recovered only employing their reflection spectra. A mechanically modeled plastic asymmetric plate has been characterized under different loading conditions using a SFBG of $N=7 \mathrm{sec}$ tions with a spatial resolution of $\Delta L=0.7 \mathrm{~mm}$. The obtained strain profiles show a very good agreement with the theoretical mechanical behavior. Using the proposed transducer, the avoidance of the compensation step has been also experimentally demonstrated just by preloading the transducer with an homogeneous strain value prior to its installation.

\section{Acknowledgment}

Special thanks to Dr. Mirapeix Serrano for his support and reviews and to Roberto Perez Sierra for his valuable collaboration with the experimental works. This work has been supported by the project TEC2010-20224-C02-02 and grant AP2009-1403.

\section{References}

[1] J.M. Lopez-Higuera. Handbook of optical fibre sensing technology. John Wiley and Sons Inc, 2002.

[2] YJ Rao, DA Jackson, L. Zhang, and I. Bennion. Strain sensing of modern composite materials with a spatial/wavelength-division multiplexed fiber grating network. Optics letters, 21(9):683-685, 1996.

[3] J.M. Lopez-Higuera, L. Rodriguez Cobo, A. Quintela Incera, and A. Cobo. Fiber optic sensors in structural health monitoring. Journal of lightwave technology, 29(4):587-608, 2011.

[4] Farhad Ansari. State-of-the-art in the applications of fiber-optic sensors to cementitious composites. Cement and Concrete Composites, 19(1):319, 1997.

[5] IJ Read and PD Foote. Sea and flight trials of optical fibre bragg grating strain sensing systems. Smart materials and structures, 10(5):1085, 2001 .

[6] Lidia Carvalho, JC C Silva, RN Nogueira, JL Pinto, HJ Kalinowski, and JA Simões. Application of bragg grating sensors in dental biomechanics. The Journal of Strain Analysis for Engineering Design, 41(6):411-416, 2006. 
[7] J. Azana and M.A. Muriel. Reconstructing arbitrary strain distributions within fiber gratings by timefrequency signal analysis. Optics letters, 25(10):698-700, 2000.

[8] X. Chapeleau, P. Casari, D. Leduc, Y. Scudeller, C. Lupi, R.L. Ny, and C. Boisrobert. Determination of strain distribution and temperature gradient profiles from phase measurements of embedded fibre bragg gratings. Journal of Optics A: Pure and Applied Optics, 8:775, 2006.

[9] M. LeBlanc, SY Huang, M. Ohn, A. Guemes, and A. Othonos. Distributed strain measurement based on a fiber bragg grating and its reflection spectrum analysis. Optics letters, 21(17):1405-1407, 1996.

[10] F. Casagrande, P. Crespi, A.M. Grassi, A. Lulli, R.P. Kenny, and M.P. Whelan. From the reflected spectrum to the properties of a fiber bragg grating: a genetic algorithm approach with application to distributed strain sensing. Applied Optics, 41(25):5238-5244, 2002.

[11] CC Cheng, Y.L. Lo, WY Li, CT Kuo, and HC Cheng. Estimations of fiber bragg grating parameters and strain gauge factor using optical spectrum and strain distribution information. Applied Optics, 46(21):4555-4562, 2007.

[12] BJ Eggleton, PA Krug, L. Poladian, and F. Ouellette. Long periodic superstructure bragg gratings in optical fibres. Electronics letters, 30(19):1620-1622, 1994.

[13] L. Rodriguez-Cobo, A. Cobo, and JM Lopez-Higuera. Sampled fiber bragg grating spectral synthesis. Optics Express, 20(20):22429-22441, 2012 .

[14] Kenneth O Hill and Gerald Meltz. Fiber bragg grating technology fundamentals and overview. Lightwave Technology, Journal of, 15(8):12631276, 1997.

[15] R. Kashyap. Fiber bragg gratings. Academic Pr, 1999.

[16] J. Kennedy and R. Eberhart. Particle swarm optimization. volume 4, pages 1942-1948 vol. 4. IEEE, 1995. 